\title{
Guest Editorial \\ Foreword to the Special Issue on Pattern Recognition in Remote Sensing
}

$\mathbf{T}$ HIS special issue is associated with the Fourth International Workshop on Pattern Recognition in Remote Sensing (PRRS 2006) that took place on August 20, 2006, in Hong Kong as part of the International Conference on Pattern Recognition (ICPR 2006). Both PRRS and ICPR are sponsored by the International Association for Pattern Recognition (IAPR). PRRS 2006 was also sponsored by the IEEE Geoscience and Remote Sensing Society. PRRS workshops are implemented by IAPR Technical Committee 7 on Remote Sensing.

The PRRS workshop has established itself as an important event for scientists who are involved in the combined fields of pattern recognition and remote sensing. These two research fields have always overlapped, but the large volumes of remote sensing data that are recently available require advanced algorithms and techniques for automatic analysis.

Papers for this special issue were solicited from participants in the PRRS workshop and from the pattern recognition, geoscience, and remote sensing communities at large through announcements in the IEEE TRANSACTIONS ON GEOSCIENCE And Remote Sensing (TGRS). Papers were sought that encompassed interdisciplinary research in the sense of applying novel pattern recognition techniques to unsolved problems in remote sensing image detection and analysis. The 14 papers included in this special issue were selected from the papers received from this solicitation using a thorough external review process.

These special issue papers describe a wide range of applications of pattern recognition techniques to problems in remote sensing image detection and analysis. They include analysis approaches for hyperspectral data, synthetic aperture radar data, LiDAR data, as well as multisensor data. The various remote sensing applications range from sea-ice detection to river flood extent delineation, individual tree detection, road tracking, and urban area detection to classifying ecoclimatic arid and semiarid zones on Earth and impact crater detection on Mars. The pattern recognition tools used come from a diverse range of techniques such as support vector machines, clustering, selforganizing maps, matched filters, template matching, artificial immune classifiers, online learning, conditional random fields, and graph-theoretic models.

We are pleased to submit this special issue to the TGRS community and hope that it accomplishes our goal of highlighting outstanding recent advances in the combined fields of pattern recognition and remote sensing.

The Fifth International Workshop on Pattern Recognition in Remote Sensing (PRRS 2008) is planned to be held in Tampa Bay, FL, in conjunction with ICPR 2008 in December. We look forward to your participation in this interdisciplinary workshop.

\section{David A. Clausi, Guest Editor}

Department of Systems Design Engineering

University of Waterloo

Waterloo, ON N2L 3G1, Canada

\section{Selim Aksoy, Guest Editor}

Department of Computer Engineering

Bilkent University

Ankara 06800, Turkey

\section{James C. Tilton, Guest Editor}

National Aeronautics and Space Administration

Goddard Space Flight Center

Computational and Information Science and Technology Office Greenbelt, MD 20771 USA 


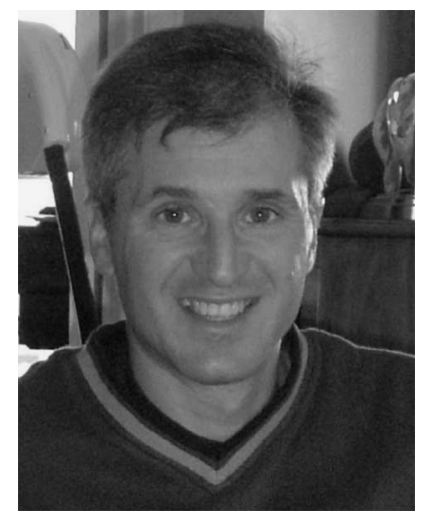

David A. Clausi (S'93-M'96-SM'03) received the B.A.Sc., M.A.Sc., and Ph.D. degrees in systems design engineering from the University of Waterloo, Waterloo, ON, Canada, in 1990, 1992, and 1996, respectively.

After completing his doctorate, he worked in the medical imaging field at Mitra Imaging Inc., Waterloo. He started his academic career in 1997 as an Assistant Professor in geomatics engineering at the University of Calgary, Calgary, AB, Canada. In 1999, he returned to the University of Waterloo and is currently an Associate Professor in the Department of Systems Design Engineering, specializing in the fields of intelligent systems, as well as societal and environmental systems. He is an active interdisciplinary and multidisciplinary researcher. He has an extensive publication record, publishing refereed journal and conference papers in the diverse fields of remote sensing, computer vision, algorithm design, and biomechanics. His primary research interest is the automated interpretation of SAR sea-ice imagery, in support of operational activities. His research efforts have led to successful commercial implementations.

Prof. Clausi was the Co-Chair of the International Association for Pattern Recognition Technical Committee 7 on Remote Sensing during 2004-2006. He has received numerous scholarships, paper awards, and two Teaching Excellence Awards.

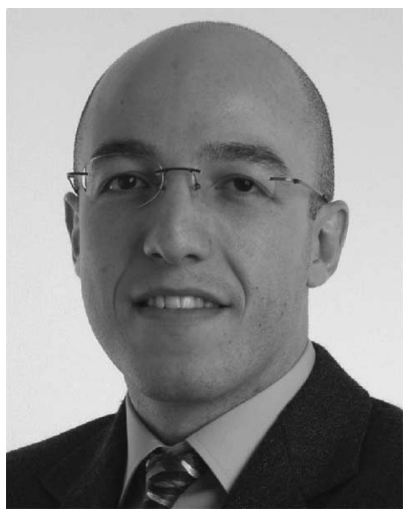

Selim Aksoy (S'96-M'01) received the B.S. degree from the Middle East Technical University, Ankara, Turkey, in 1996 and the M.S. and Ph.D. degrees from the University of Washington, Seattle, in 1998 and 2001, respectively.

He has been an Assistant Professor at the Department of Computer Engineering, Bilkent University, Ankara, since 2004, where he is also the Co-Director of the RETINA Vision and Learning Group. Before joining Bilkent, he was a Research Scientist at Insightful Corporation, Seattle, where he was involved in image understanding and data mining research sponsored by the National Aeronautics and Space Administration, the U.S. Army, and the National Institutes of Health. During 1996-2001, he was a Research Assistant at the University of Washington, where he developed algorithms for content-based image retrieval, statistical pattern recognition, object recognition, graph-theoretic clustering, user relevance feedback, and mathematical morphology. During the summers of 1998 and 1999, he was a Visiting Researcher at the Tampere International Center for Signal Processing, Tampere, Finland, collaborating in a content-based multimedia retrieval project. His research interests include computer vision, statistical and structural pattern recognition, machine learning and data mining with applications to remote sensing, medical imaging, and multimedia data analysis.

Dr. Aksoy is a member of the International Association for Pattern Recognition (IAPR). He received the CAREER Award from the Scientific and Technical Research Council of Turkey (TUBITAK) in 2004. He served as the Vice Chair of the IAPR Technical Committee 7 on Remote Sensing during 2004-2006 and is currently the Chair of the same committee for 2006-2008.

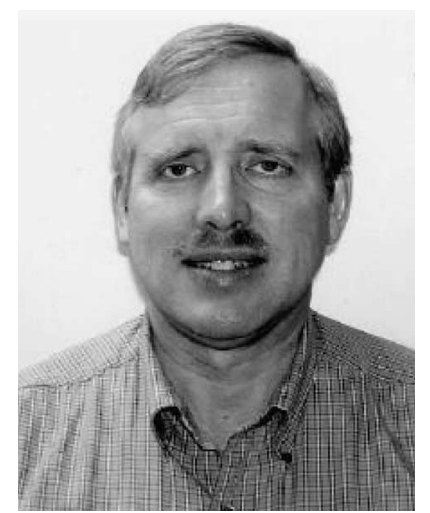

James C. Tilton (S'79-M'81-SM'94) received the B.A. degrees in electrical engineering, environmental science and engineering, and anthropology and the M.E.E. degree in electrical engineering from Rice University, Houston, TX, in 1976, the M.S. degree in optical sciences from the University of Arizona, Tucson, in 1978, and the Ph.D. degree in electrical engineering from Purdue University, West Lafayette, IN, in 1981.

He is currently a Computer Engineer with the Computational and Information Science and Technology Office (CISTO), Goddard Space Flight Center, National Aeronautics and Space Administration (NASA), Greenbelt, MD. He was with Computer Sciences Corporation from 1982 to 1983 and with Science Applications Research from 1983 to 1985 on contracts with the NASA Goddard Space Flight Center. As a member of CISTO, he is responsible for designing and developing computer software tools for space and Earth science image analysis and encouraging the use of these computer tools through interactions with space and Earth scientists. His recursive implementation of hierarchical segmentation algorithm (RHSEG) has resulted in one patent and an additional patent application. His latest project is the integration of a knowledge discovery system with image segmentation hierarchies produced by RHSEG.

Dr. Tilton is a member of the IEEE Geoscience and Remote Sensing Society (GRSS) and the IEEE Signal Processing Society. He is also a Member of Phi Beta Kappa, Tau Beta Pi, and Sigma Xi. From 1992 through 1996, he served as a member of the IEEE GRSS Administrative Committee. Since 1996, he has served as an Associate Editor for the IEEE TRANSACTIONS ON Geoscience AND REMote SENSING. He has been the Chair of the Washington/Northern Virginia chapter of the IEEE GRSS since 2004. 